\title{
SOME THEOREMS ON MEROMORPHIC FUNCTIONS
}

S. M. SHAH

1. Introduction. In a recent paper [1 $]^{1}$ Yoshitomo Okada proved the following two theorems.

THEOREM A. If for any meromorphic function

$$
F(z)=f(z) / g(z),
$$

where $f$ and $g$ are canonical products of genera $p, q$ and of orders $\rho_{1}, \rho_{2}$ respectively,

$$
\max \left(\rho_{1}, \rho_{2}\right)=\max (p, q),
$$

then

$$
\liminf _{r \rightarrow \infty} \frac{1}{r N(r) \phi(r)} \int_{0}^{r} \log ^{+} M(t, F) d t=0,
$$

where $N(r)=n(r, f)+n(r, g)$ and $\phi(x)$ is any positive continuous nondecreasing function of a real variable $x$ such that $\int_{a}^{\infty} d x / x \phi(x)$ is convergent.

THEOREM B. If (1) is a function of order $\rho$, where $\rho>0$ is not an integer, then

$$
\liminf _{r \rightarrow \infty} \frac{1}{r N(r)} \int_{0}^{r} \log ^{+} M(t, F) d t<\infty .
$$

In this paper we extend Theorems A and B. Let

$$
F(z)=z^{k} \exp (H(z)) f(z) / g(z)
$$

be any meromorphic function of finite order $\rho$. Here $H(z)$ is a polynomial of degree $h ; f(z)$ and $g(z)$ are canonical products of orders $\rho_{1}, \rho_{2}$ and genera $p, q$ respectively. The genus of $F(z)$ is $P=\max (p, q, h)$ and we have $\rho-1 \leqq P \leqq \rho$. Let $n(r, 0)$ and $n(r, \infty)$ denote the number of zeros and poles respectively of $F(z)$ in $|z| \leqq r$ and write $\psi(r)$ $=n(r, f)+n(r, g)$,

$$
I(r, F)=I(r)=\frac{1}{r} \int_{0}^{r} \log ^{+} M(t, F) d t .
$$

THEOREM 1. If for any meromorphic function (5) of order $\rho$ where

Received by the editors September 13, 1950.

${ }^{1}$ Numbers in brackets refer to the references at the end of the paper. 
$\rho>0$ is an integer,

$$
h \leqq \max (p, q)=s(s a y),
$$

then

$$
\underset{r \rightarrow \infty}{\lim \inf } I(r, F) /\{n(r, 0)+n(r, \infty)\} \phi(r)=0,
$$

where $\phi(x)$ has been defined in the statement of Theorem A.

ThEOREM 2. For any meromorphic function (5) of order $\rho$ where $\rho>0$ is not an integer, we have

$$
\liminf _{r \rightarrow \infty} I(r, F) /\{n(r, 0)+n(r, \infty)\}<\infty .
$$

THEOREM 3. If the meromorphic function (5) be nonconstant and of zero order, then

$$
\lim \inf I(r, F) /\{N(r, 0)+N(r, \infty)\}<\infty,
$$

where $N(r, a)$ denotes as usual

$$
\int_{0}^{r} \frac{n(x, a)-n(0, a)}{x} d x+n(0, a) \log r .
$$

Corollary.

$$
\liminf _{r \rightarrow \infty} I(r, F) /\{n(r, 0)+n(r, \infty)\} \log r<\infty .
$$

2. Examples. If $h>\max (p, q)$, then (7) does not hold. For instance, if

$$
F(z)=e^{z} \prod_{2}^{\infty}\left\{1+\frac{z}{n(\log n)^{\alpha}}\right\}, \quad \alpha>1,
$$

then $h=1, \max p, q)=0$, and

$$
\frac{I(r)}{\{n(r, 0)+n(r, \infty)\} \log r(\log \log r)^{2}} \rightarrow \infty,
$$

as $r \rightarrow \infty$. Further let $\alpha(x)$ be any given function tending to infinity, however slowly, with $x$ and consider

$$
F(z)=\prod_{2}^{\infty} E\left(\frac{z}{\alpha_{\nu}}, p\right),
$$

where 


$$
\alpha_{\nu}=-\left\{\nu(\log \nu)^{\alpha}\right\}^{1 / \rho}, \quad p<\rho<p+1 ;
$$

then $F(z)$ is an entire function of nonintegral order $\rho>0$ and we have $[5$, p. 44$]$

$$
\lim _{r \rightarrow \infty} \frac{I(r, F) \alpha(r)}{\{n(r, 0)+n(r, \infty)\}}=\infty .
$$

Further $F(z)=\prod_{n=1}^{\infty}\left(1-z / e^{n}\right)$ is an entire function of zero order for which

$$
\begin{gathered}
\lim _{r \rightarrow \infty} \frac{I(r, F) \alpha(r)}{\{N(r, 0)+N(r, \infty)\}}=\infty, \\
\lim _{r \rightarrow \infty} \frac{I(r, F)}{\{n(r, 0)+n(r, \infty)\} \log r}=\frac{1}{2} .
\end{gathered}
$$

3. LemMA. Let

$$
J(r, p)=\int_{0}^{\infty} \frac{r^{p+1} \psi(t) d t}{t^{p+1}(t+r)} .
$$

If $s \geqq h$ and $\psi(t) \geqq 1$ for all large $t$, then ${ }^{2}$

$$
I(r, F)<H J(r, s) \text {. }
$$

Proof. Let $\left(a_{n}\right)_{1}^{\infty}$ denote the zeros of $f(z)$ and $\left(b_{n}\right)_{1}^{\infty}$ the zeros of $g(z)$ and let $k>1$. Then

$$
\begin{aligned}
T(r, F)< & T(r, f)+T(r, g)+O\left(r^{h}+\log r\right) . \\
I(r, F)< & H T(k r, F)<H\{T(k r, f)+T(k r, g)\}+O\left(r^{h}+\log r\right) \\
< & H\left\{\log ^{+} M(k r, f)+\log ^{+} M(k r, g)\right\}+O\left(r^{h}+\log r\right) \\
< & H\left\{\sum_{n=1}^{\infty} \frac{r^{s+1}}{\left|a_{n}\right|^{s}\left(r+\left|a_{n}\right|\right)}+\sum_{1}^{\infty} \frac{r^{s+1}}{\left|b_{n}\right|^{s}\left(r+\left|b_{n}\right|\right)}\right\} \\
& +O\left(r^{h}+\log r\right) \\
< & H \int_{0}^{\infty} \frac{r^{s+1} \psi(t) d t}{t^{s+1}(t+r)}+O\left(r^{h}+\log r\right) .
\end{aligned}
$$

Now

$$
J(r, s)>\frac{1}{2} \int_{0}^{r} \frac{r^{s} \psi(t) d t}{t^{s+1}}>h_{1} r^{s} .
$$

Hence if $s>0, I(r)<H J(r, s)$. If $s=0$ then $h=0$ and since $\psi(t) \geqq 1$ for all large $t$,

${ }^{2} H\left(h_{1}\right)$ denotes a positive constant not necessarily the same at each occurrence. 


$$
\int_{0}^{r} \frac{\psi(t)}{t} d t>h_{1} \log r
$$

which proves the lemma.

4. Proof of Theorem 1. We note that $\psi(r) \geqq 1$ for all large $r$, for if $\psi(r)=0$ for all $r$, then $s=0$ and hence, by (6), $h=0$ and $F(z)$ would then not be a function of order greater than or equal to one.

Consider $G(z)=\prod_{1}^{\infty} E\left(z / c_{n}, s\right)$, where the sequence $c_{1}, c_{2}, \cdots$ is composed of $a_{1}, a_{2}, \cdots ; b_{1}, b_{2}, \cdots$ and $\left|c_{1}\right| \leqq\left|c_{2}\right| \leqq \cdots$. Since $h \leqq s, G(z)$ is an entire function of order $\rho$ and genus $s=\max (p, q)$. Further $s=\rho$ or $\rho-1$ and hence we have $[2$, pp. 23-29;3, pp. 180-186]

$$
\liminf _{r \rightarrow \infty} \frac{J(r, s)}{\psi(r) \phi(r)}=0 \text {. }
$$

Since

$$
\psi(r) \leqq n(r, 0)+n(r, \infty),
$$

(7) follows from the lemma.

5. Proof of Theorem 2. This theorem follows from the argument of Okada $[1$, p. 249$]$. We sketch an alternative proof. Let $[\rho]=P$. Then $h \leqq P=s$. Let $0<\epsilon<\min \{\rho-s, s+1-\rho\}$. From the lemma we have

$$
I(r, F)<B\left\{\int_{0}^{r} \frac{r^{s} \psi(t) d t}{t^{s+1}}+r^{s+1} \int_{r}^{\infty} \frac{\psi(t) d t}{t^{s+2}}\right\}
$$

From Lemma $3[3$, p. 184] we have

$$
\frac{\psi(t)}{t^{\rho-\epsilon}} \leqq \frac{\psi\left(r_{n}\right)}{r_{n}^{p-\epsilon}}, \quad 0 \leqq t \leqq r_{n} ; \quad \frac{\psi(t)}{t^{p+e}} \leqq \frac{\psi\left(r_{n}\right)}{r_{n}^{p+\epsilon}}, \quad t \geqq r_{n},
$$

for a sequence $\left(r_{n}\right)_{1}^{\infty}, r_{n} \uparrow \infty$. Hence the theorem follows.

6. Proof of Theorem 3. If $\psi(t)=0$ for all $t$ then $F(z)$ would be of the form $A z^{k}$ and (9) and (10) obviously hold. Hence we may suppose that $\psi(t) \geqq 1$ for all large $t$. Let

$$
\psi_{1}(t)=\int_{0}^{r} \frac{\psi(t)}{t} d t
$$

Then

$$
I(r)<B\left[\int_{0}^{r} \frac{\psi(t)}{t} d t+r \int_{r}^{\infty} \frac{\psi(t)}{t^{2}} d t\right]=H r \int_{r}^{\infty} \frac{\psi_{1}(t)}{t^{2}} d t
$$


Now

$$
\lim _{r \rightarrow \infty} \frac{\psi_{1}(r)}{r^{e}}=0 .
$$

Hence there exists a sequence $\left\{r_{n}\right\}_{1}^{\infty}, r_{n} \uparrow \infty$, such that

$$
\frac{\psi_{1}(r)}{r^{e}} \leqq \frac{\psi_{1}\left(r_{n}\right)}{r_{n}^{e}} \quad \text { for } r \geqq r_{n} ;
$$

and the theorem follows. The corollary follows directly from the theorem.

\section{REFERENCES}

1. Yoshitomo Okada, Some theorems on meromorphic functions, Proceedings of the American Mathematical Society vol. 1 (1950) pp. 246-249.

2. S. M. Shah, $A$ theorem on integral functions of integral order, J. London Math. Soc. vol. 15 (1940) pp. 23-31.

3. - A theorem on integral functions of integral order (II), J. Indian Math. Soc. vol. 5 (1941) pp. 179-188.

4. - A note on meromorphic functions, The Mathematics Student vol. 12 (1944) pp. 67-70.

5. R. Nevanlinna, Le theorème de Picard-Borel et la theorie des fonctions meromorphes, Paris, 1929.

Aligarh University 